\title{
SCIENTIFIC APPROACHES TO THE DEVELOPMENT OF WOUND-HEALING SOFT DRUGS FOR THE TREATMENT OF SERVICEMEN AT THE HOSPITAL STAGE
}

\author{
Tarasenko V. O., Candidate of Pharmaceutical Sciences, Associate Professor, Ukrainian Military \\ Medical Academy, Kyiv, Ukraine, ORCID ID: https://orcid.org/0000-0002-3614-6752 \\ Drozdov D. V., Candidate of Pharmaceutical Sciences, Associate Professor, Ukrainian Military \\ Medical Academy, Kyiv, Ukraine, ORCID ID: https://orcid.org/0000-0002-7769-750X \\ Bielozorova O. V., Candidate of Pharmaceutical Sciences, Head of the Scientific and Organizational \\ Department, Ukrainian Military Medical Academy, Kyiv, Ukraine, \\ ORCID ID: https://orcid.org/0000-0001-7257-3965 \\ Plieshkova O. V., Senior Lecturer, Ukrainian Military Medical Academy, Kyiv, Ukraine, \\ ORCID ID: https://orcid.org/0000-0002-9000-7358 \\ Dobrovolnyi O. O., Professor, Ukrainian Military Medical Academy, Kyiv, Ukraine, \\ ORCID ID: https://orcid.org/0000-0002-9835-3909
}

\section{DOI: https://doi.org/10.31435/rsglobal_conf/30032021/7475}

In the course of the Anti-Terrorist Operation in Ukraine, gunshot and combined wounds dominate in the structure of sanitary casualties among the combatants (around 86.9\%); two thirds of these are limb wounds, combined wounds and head wounds, all of which require urgent and high quality medical treatment, including use of modern highly-efficient wound-healing preparations. Limb wounds which account for 53\% dominate localization of patients'groups, and require urgent elimination of the development of the microflora in the wound, creating optimum conditions for the wound process to develop into the regeneration phase [3, 7]. Nowadays the practice of treating wounds under dressings makes possible a significant shortening of the systematic antibacterial therapy period, by searching and active introduction of new medical preparations with poly-factor effect and capable of mutuallypotentiating and mutually supporting activity, which meet the current requirements of pharmaceutical safety and allow efficient local treatment of wounds in accordance with the stage of the wound process [1, 8]. The importance of developing wound-healing preparations with complex effects is conditioned by the increase in the number of post-operational infectious complications, resistance of microorganisms to antibiotics, changes in immune-biological responsiveness of the macroorganism, etc. [3, 6].

The search for active substances and adjuvants and methods of technology of production with consideration of physiological peculiarities of the wound surface are the main directions in the development of biopharmaceutics in the sphere of development and enlargement of the nomenclature of domestic soft medical preparations for treatment of the wound process [12]. Currently, vulnerary medical preparations are one of the most common pharmaceutical forms. They make up $10 \%$ of the total output of the production of ready to use formulations. Soft pharmaceutical preparations are used in different spheres of medicine for treatment, diagnosis and prevention of diseases (Swarbrick, 2013). They are characterized by relative simplicity and safety of usage, economical and technological effectiveness [13]. The regulation of pharmaceutical changing factors allows modification of the properties of pharmaceutical ingredients, facilitating their distribution on the wound surface, and prolonging their therapeutic effect, etc. [14]. The contact method of implementation of soft pharmaceutical preparations for treating purulent-inflammatory processes allows maximum concentration of the medical substance on the wound area. The method of soft medication introduction is considered the safest, for the greater part of the dose is on the surface of the wounded area $[7,15]$.

The aim of this article is to present research focused on the scientific grounding and development of a modern Ukrainian-made medical preparation with a complex effect for local treatment of wounds, in the form of an ointment, capable of anti-bacterial, anti-inflammatory and local anesthetic effect, and which does not require frequent changes in the treatment of wounded military personnel.

Materials and methods. Technological, physical-chemical and biopharmaceutical indicators of the ointment were calculated according to the generally accepted methods in accordance with the National Pharmacopoeia of Ukraine [9]. 
The pharmacotherapeutic effect of medical preparations for treating purulent-inflammatory processes is closely related to the nature, properties and quantitative ratios of the base components. Rationally selected components of the base can increase the therapeutic activity of the active pharmaceutical ingredients, support or impede its release and absorption, increase or decrease its pharmaceutical effect, affect its bioavailability and define various sideeffects, distinguishing the effect of the preparation (resorptive or surface [4]. The base is essential to the pharmacotherapeutic effect and pharmacokinetics of the preparation.

The base impacts the state, properties and the pathological process of the wounded area covered by the soft pharmaceutical preparation. Also, the base determines the consumer properties of the soft pharmaceutical [14]. Currently, pharmaceutical practice includes around 200 individual and combined ointment and gel bases, which possess different properties and correspond to different medical-biological purposes.

There is no perfect base, for provision of the base with required properties involves combining several adjuvants $[4,10]$. As we know, ointments, creams for treating purulent-inflammatory processes, should at the same time possess multi-directional impact on the main etiopathogenetic factors of the inflammation, sufficient osmotic activity, preventing secondary infection [8]. Medical usage of the preparation is defined by the usage of the corresponding adjuvants and bases.

In treatment of purulent wounds it is necessary to consider that over the purulent-necrotic phase of the wound process, it is recommended to use soft pharmaceutical forms with a strong antimicrobial, anti-inflammatory, anesthetic and osmolar effect, which are capable of controllable dehydrating activity and impact on the release, bioavailability and therapeutic effect of medical substances [4, 10]. It is generally accepted that the manifestation of osmotic activity in antiinflammatory preparations promotes dehydration in the zone of inflammation, which leads to decrease of edemas and accelerates the metabolic processes in the tissues [9].

The substances having these properties are polyethylene oxides of the base (from mixture of macrogols), which due to their bacteriostatic properties are resistant to microbial contamination, and also fully release medical substances and prolong their effectiveness $[9,10]$. Bioavailability of the active pharmaceutic ingredients is not related to the temperature of the melting of polyethylene oxidal base and is conditioned only by the speed of dissolution of the bases and their diffusion. From the technological perspective, the advantage of polyethylene oxide (PEO) bases is high viscosity, which prevents the sedimentation of non-dissolved medical substances [11, 15]. Chemical indifference, thermal stability, absence of polymorphic modifications and resistibility to $\mathrm{pH}$ changes determine the suitability of polyethylene oxide bases for production of many pharmaceutical forms for treating purulent-inflammatory processes. The high affinity of the base to biological fluids in the organism (interstitial fluid) facilitates the full release of the active pharmaceutical ingredients from the pharmaceutical form over a certain period of time [14]. Considering the requirements of woundtreatment preparations, the usage of hydrophobic bases is not appropriate, for they minimize the dynamic processes of absorption, and, therefore decrease penetration and release of the medical substances [11]. Modern production of soft pharmaceutical forms for treating purulent-inflammatory processes prefers pharmaceutical preparations on hydrophilic bases, which can be applied to the wound surface without disturbing the perspiration. Active pharmaceutical ingredients are easily absorbed from such [4]; non-aqueous solvents in the base, which affect the penetrability of the cellular membranes and increase the absorbability of the preparation, contribute to this process.

Therefore, use of hydrophilic or combined bases which are highly effective in releasing active pharmaceutical ingredients is optimal for developing wound-treatment preparations. This is due to the fact that achieving optimum therapeutic effect in the wounded area requires mixing the base and the medical preparation with exudates of damaged tissues $[7,8]$. Pharmaceutical practice uses bases with elasticviscous flexible environment (emulsion of the I generation) and hydrophilic non-aqueous solvents and carbomer gels on the base of hydrophilic surfactants.

A no less important characteristic of the development of woundtreatment preparations is considered to be the use of I generation emulsion bases (oil/water), which are effective in contacting the wound surface, are capable of a cooling effect due to active evaporation of the water phase of the base, and are able to provide an anesthetic and soothing effect to a certain extent [5]. 
Recent biopharmaceutical studies on pharmaceutical preparations of local application have shown that with a well reasoned selection of the base, it is possible to provide a strong and sometimes intensified effect of the medical substances included in the compound. The authors introduced new polyethylenebased ointments (combinations of polyethylene oxides with molecular weight of 400 and 1500) into clinical practice. Polyethylene oxides are of low toxicity and express clearly visible osmotic properties [4].

Kadajji and Betageri [10] emphasize that polyethylene - based combined ointments are highly promising, being different from traditional preparations due, first of all, to their multidirectional effect. In particular, these preparations allow management of the intensity and the direction of the diffusion process after applying the formulation to the wound for differential usage at different stages of the wound process. The authors consider [12] that intensified efficiency of the prevention and treatment of the infectious complications in wounds is currently impossible without radically new combined ointments on modern bases, which include highly efficient antibacterial preparations: Levomycetin (Levosin, Levomecol), Dioxydin (5\% Dioxydin ointment, Dioxycolum), Nltazolum (Nitacid) [2]. Also, the new ointments include such preparations as trimecaine hydrochloride for providing an anesthetic effect of the ointment and methyluracil, which is capable of anabolic and anti-catabolic activity for stimulating processes of cell. In our research, for achieving optimum pharmaceutical effect, particularly antibacterial, anti-inflammatory, and analgesic effects, the compound of the studied ointment included active pharmaceutical ingredients, which are commonly used and which proved to work well in dermatological and surgical practice: methyluracil $\mathrm{CO}_{2}$ extract of chamomile, anaesthesin [2].

Choice of hydrophilic non-aqueous solvents in the cream. A number of authors [2,9] mention glycerine as one of the most common hydrophilic non-aqueous solvents in production of pharmaceutical formulations of local application. It is able to affect the main technological and biopharmaceutical characteristics of a preparation. Also, glycerine is recognized as a classic moisturizing and softening pharmaceutical agent and is capable of being a penetrant and a solubilizer. When it is applied to the skin, this compound absorbs water from the subepidermal layers, forming a hydrogen bond with it. Therefore, it prevents the loss of moisture by keeping the water in the derma. Another significant peculiarity of glycerine is that its molecules structure the extracellular fluid and protect the cells against damage from excessively high osmotic impact. Moreover, glycerine increases transepidermal penetration of water and other substances [14, 15]. Like the other hydrophilic nonaqueous solvents, glycerine influences the frost-resistance and osmotic properties of the base, and also the absorption of active pharmaceutical ingredients.

Introducing glycerine to the compound of the developed pharmaceutical form was mostly conditioned by the necessity to heighten the moisturizing and softening properties of the base. These indicators were the main criteria. Also, the inclusion of glycerine in the proposed composition positively affects the following characteristics of the base: viscosity and other rheological indicators, speed of absorption of active pharmaceutical ingredients; adhesion on application; osmotic properties.

It should be mentioned that when using glycerine included in the ointment base, the phase of active osmosis is followed by the phase of "reverse osmosis". This solvent is capable of a penetrating effect, which allows its molecules enter the aqueous environment through the membrane.

Due to these properties, glycerine allows the creation of ointment bases with a prolonged but a mild dehydrating effect $[4,14]$. According to the authors, it is recommended that cosmetic and pharmaceutical preparations for external application should include glycerine as an active moisturizing component in the amount of up to $15 \%$. At higher content, surplus glycerine forms a non-drying membrane on the skin. Generally, for providing moisturizing and alleviating properties, glycerine is used in concentrations of $5 \%$ or $10 \%$ [3].

Conclusions. Summarizing the accumulated experience in the treatment of wounds and current knowledge of biopharmaceutical aspects of the creation of soft drugs, it is concluded that successful prevention and rapid treatment of the specified pathological condition is possible with a comprehensive approach and use of common measures. Significantly increase the effectiveness of treatment of wound infection will allow the use of the basis developed by the authors for the combination cream, the composition of which is scientifically substantiated according to the pathogenesis of the wound process, taking into account its phase and nature of the microflora.

Current biopharmaceutical studies of topical medicinal products have proven that, with reasonable choice of carrier, it is possible to provide a pronounced, and sometimes enhanced, effect of 
the drugs introduced into its composition. Physico-chemical studies have justified the optimal carrier for ointment - PEO-1500 and PEO-400 (1:4), whose osmotic properties ensure the elimination of purulent discharge. PEO-400, forming complexes with antimicrobial compounds, transports them to the depth of damaged tissues - the main sites of microbial localization, and PEO-1500 provides a uniform and long-term absorption of exudate.

Structural-mechanical studies have proved the concentration of emulsifier № 1 in the amount of 3,2\%.

Determination of structural and mechanical properties of the cream base indicate that it belongs to structured systems, has thixotropic properties, which causes good consumer (ease and ease of application) and technological (packing) properties.

The results of biopharmaceutical research on the development of the basis of cream for the treatment of wounds may be the basis for the development of the cream and the conduct of clinical research and introduction into industrial mass production.

\section{REFERENCES}

1. Atiyeh, B. S., Dibo, S. A. \& Hayek, S. N. (2009). Wound cleansing, topical antiseptics and wound healing. International Wound Journal, 6(6), 420-430.

2. Barajas-Nava, L. A., López-Alcalde, J., Roqué i Figuls, M., Solà, I. \& Bonfill, Cosp X. (2013). Antibiotic prophylaxis for preventing burn wound infection. Cochrane Database of Systematic Reviews, 6.

3. Cardinal, M. M., Eisenbud, D. E. \& Armstrong, D. G. (2009). Serial surgical debridement: a retrospective study on clinical outcomes in chronic lower extremity wounds. Wound Repair and Regeneration, 17(3), 306-311.

4. Chan, B. A., Sunting. X., Li, A., Simpson, J. M., Sternhagen., G, L., Yu, T., Darvish, O. A., Naisheng, J. \& Donghui, Z. (2017). Polypeptid polymers: synthesis, characterization, and properties. Biopolymers.

5. Debjit, B., Harish, G., B. Pragati, K. S., Duraivel, K. P. \& Sampath, K. (2012). Recept advances in novel topical drug delivery system. The Pharma Innovation, 1 (9), 12-31.

6. Dohmen, P. M. (2008). Antibiotic resistanse in common pathogens reinforces the need to minimize surgical site infections. Journal of Hospital Infection, 70(2), 15-20.

7. Healy, B. M. \& Freedman, A. M. (2010). ABC of wound healing Infections. British Medical Journal, 332, 838-841.

8. Hotz, B. V., Visekruna, A. A., Buhr, H. J. \& Hotz H. G. (2010). Beyond epithelial to mesenchymal transition: a novel role for the transcription factor snail in inflammation and wound healing. World Journal of Gastrointestinal Surgery, 14(2), 388-397.

9. Hryzodub, O. I. (ed.), (2015). Derzhavna farmakopeya Ukrayiny [State pharma-copoeia of Ukraine]. Ukrayins'kiy Naukoviy Farmakopeyniy Tsentr Yakosti Likars'kykh Zasobiv, Kharkiv (in Ukrainian).

10. Kadajji, V. G. \& Betageri, G. V. (2011). Water soluble polymers for pharmaceutical Applications. Polymers, 3(4), 1972-2009.

11. Maitz, M. F. (2015). Applications of synthetic polymers in clinical medicine. Biosurface and Biotribology, 1(3).

12. Merkle, H. P. (2015). Drug delivery's quest for polymers: where are the frontiers? European Journal Pharmaceutical Biopharmaceutics, 97, 293-303.

13. Owens, C. D. \& Stoessel, K. P. (2008). Surgical site infections: epidemiology, microbiology and prevention. Journal of Hospital Infection, 70(2), 3-10.

14. Sheskey, P. J., Cook, W. G. \& Cable, C. G. (2017). Handbook of pharmaceutical excipients. London: APhA/Pharmaceutical Press, 2017.

15. Swarbrick, J. (2013). Encyclopedia of pharmaceutical science and technology. Fourth Edition, Six Volume Set Dasel Marcel Dekker, New York. 